\title{
CARDIOLIPIN ANTIGEN*
}

\author{
III. AN EXAMINATION OF SPECIFICITY \\ BY \\ HENNING SCHMIDT
}

\author{
Statens Seruminstitut, Copenhagen. Director: Dr. J. Ørskov
}

Most authors agree that the sensitivity of cardiolipin antigen is equal to that of the unpurified antigens, while the specificity of cardiolipin antigen is appreciably higher than that of unpurified antigens (Rein and Kent, 1947 ; Vogelsang, 1948 ; Schmidt, 1951; Reyn and Schmidt, 1951; Schmidt and Reyn, 1952). Price and Wilkinson (1952), however, reported that with cardiolipin antigen they found more non-specific positive reactions in routine tests than with unpurified antigen.

Various explanations may be offered of nonspecific positive reactions, but there are three main causes :

(i) technical errors,

(ii) syphiloid reactions,

(iii) "genuine" biologically false positive reactions.

(i) Technical Errors.-These may be due to admixture to the blood sample of various chemicals such as citrate, alcohol, ether, iodine tincture, arsphenamine, tannic acid from cork stoppers, etc. (Meinicke, 1952). Maladjustment of antigen, complement, etc. must also be considered as technical errors.

(ii) "Syphiloid Reactions".-This name has been applied to the positive reactions occurring in connexion with certain spirochaetal diseases (such as yaws, pinta, and bejel) by several authors, who wished to indicate that the positive reactions occurring in such diseases are not really non-specific, because the causal spirochaetae are likely to have certain antigenic properties in common with Spirochaeta pallida, thus causing cross-reactions with the syphilitic antigens. Turner (1952) demonstrated that 4 months after injection with Nichols' pathogenic spirochaetae, rabbits were immune to challenge infections with other strains of spirochaetae recently isolated from venereal syphilis, and, in some degree, also to infection with bejel and yaws.

(iii) "Genuine" Biologically False Positive SeroReactions.-These occur in many different conditions. They may be connected with an acute disease and then disappear gradually after recovery ; in such cases they may be described as acute. In other cases the positive reaction may persist for years or for life, and may then be described as chronic.

\footnotetext{
*Received for publication October 3, 1952.
}

Biologically false positive sero-reactions (B.F.P.) may occur in a number of different conditions. Hecht (1952) mentioned malaria, diseases of the respiratory tract, pregnancy, and acute febrile diseases. Many authors emphasize the occurrence of B.F.P. in diseases of the respiratory tract (Eldh, 1932 ; Krag and Lønberg, 1938 ; Lindau, 1939 ; Davis, 1944 ; Rein and Elsberg, 1944 ; Wiingaard, 1948 ; Vogelsang, 1948), while others state that the incidence of B.F.P. should not be higher in pregnant women than among "normal" patients. (Penttinen, 1947; Krag and Røjel, 1947 ; Schmidt and Reyn, 1952).

The purpose of the present study was to investigate whether there was any difference in specificity between Wassermann's complement-fixation test as modified by Mørch, carried out with unpurified antigen (WR-M), and with cardiolipin (C-WR-M). At the same time the specificity of Kahn's standard test (KR) was examined and compared with WR-M and C-WR-M. Patients with diseases of the respiratory tract and pregnant women form the two principal groups thus examined. Under the Pregnancy Act (of October 1, 1945) all pregnant women are entitled to a serological examination for syphilis ; pregnant women, therefore, represent the largest uniform group of serologically examined persons in Denmark.

The strength of the positive sera is indicated in degrees of potency (Schmidt, 1951). However, the present study is mainly qualitative.

In this paper the findings are expressed as the " non-specificity" of the individual sero-reactions. By " non-specificity" is meant the number of positive sero-reactions in non-syphilitic patients expressed as a percentage of the total number of non-syphilitic patients examined.

\section{Material and Methods}

This examination is based on three sets of material. Only one sample is included from each examined patient with B.F.P., viz. the first positive blood sample submitted during the test period. Thus, the number of examined positive sera is equal to the number of examined patients. 
(a) 3,980 sera were examined by parallel WR-M, C-WR-M, and KR tests. Sera were chosen at random from hospital wards and out-patient departments. About 200 sera were examined each day for a 20-day period. The findings from the examination of syphilitic sera from this material have been published previously (Schmidt, 1952).

(b) 1,986 sera from pregnant women (irrespective of periods of pregnancy) were examined by parallel WR-M, C-WR-M, and KR. tests.

(c) All sera submitted to the Sero-diagnostic Department of the Statens Seruminstitut during the period November 1, 1951, to March 19, 1952, were examined by WR-M and KR, but if WR-M and or KR showed positive reactions in screen tests, such sera were examined quantitatively by C-WR-M simultaneously with the quantitative examination by WR-M and KR. This material will not reveal the sera which might have been positive in the C-WR-M test only, but it is possible, on the basis of $(a)$ and $(b)$, to estimate the magnitude of that figure.

\section{Author's Investigations}

(a) Out of the 3,980 samples examined, 3,565 sera came from patients with neither clinical evidence nor history of syphilis. Of these patients, 24 were biologically false positive reactors by one or more tests, corresponding to a non-specificity of about 0.7 per cent. (Table I).

TABLE I

DISTRIBUTION OF 24 BIOLOGICALLY FALSE POSITIVE SERA OVER THE DIFFERENT COMBINATIONS OF REACTIONS

\begin{tabular}{c|c|c|c}
\hline WR-M & C-WR-M & KR & Total \\
\hline$-E$ & + & \pm & 3 \\
\pm & \pm & \pm & 3 \\
\pm & \pm & \pm & 13 \\
- & \pm & \pm & 0 \\
\hline
\end{tabular}

The non-specificity of the individual tests was : WR-M 0.6 per cent., C-WR-M $0 \cdot 2$ per cent., KR $0 \cdot 2$ per cent.
WR-M gave the only positive reaction in thirteen cases, KR in two, and C-WR-M in one.

By omitting the WR-M, thirteen of the biologically false positive sera would thus have been avoided. One-third of the positive sera examined originated from patients with diseases of the respiratory tract. The non-specificity $(0.7$ per cent.) observed in $(a)$ is significantly higher $(P<0 \cdot 1 \text { per cent. })^{*}$ than the non-specificity of 0.3 per cent. found by Wiingaard (1948) in an examination of 232,000 non-syphilitic patients. This difference may be accounted for partly by the somewhat different sensitivity of the tests used, partly by the fact that the present material was collected from February to April, in which season diseases of the respiratory tract are more frequent than during the rest of the year. Finally, this material mainly originates from patients in hospital wards, and other authors (Bang and Krag, 1943 ; Wiingaard, 1948) have found non-specificity in such patients to be higher than in " normal" persons.

In order to ascertain significant differences, if any, between the incidence of positive sero-reactions with the various tests used in $(a)$, the 24 non-specific positive sera have been tabulated in " $2 \times 2$ Tables," one for each of the three possible combinations (Table II).

In this material WR-M is significantly more frequently positive than both C-WR-M and KR ( $P<0.1$ and 0.2 per cent. respectively), which means that WR-M is significantly more nonspecific than the two other tests. There is no difference in the incidence of C-WR-M + and $\mathrm{KR}+$. From the examinations performed for (a), the following conclusions may be drawn :

(1) The non-specificity of the examined reactions is 0.7 per cent.

(2) $\mathrm{WR}-\mathrm{M}>\mathrm{KR}=\mathrm{C}-\mathrm{WR}-\mathrm{M} . \dagger$

\footnotetext{
* The limit of significance was fixed at $P \ll 5$ per cent.

$\dagger>$ significantly greater than

$=$ not significantly different from
}

TABLE II

“ $2 \times 2$ TABLES” FOR 24 BIOLOGICALLY FALSE POSITIVE SERA FROM 3,565 NON-SYPHILITIC PATIENTS

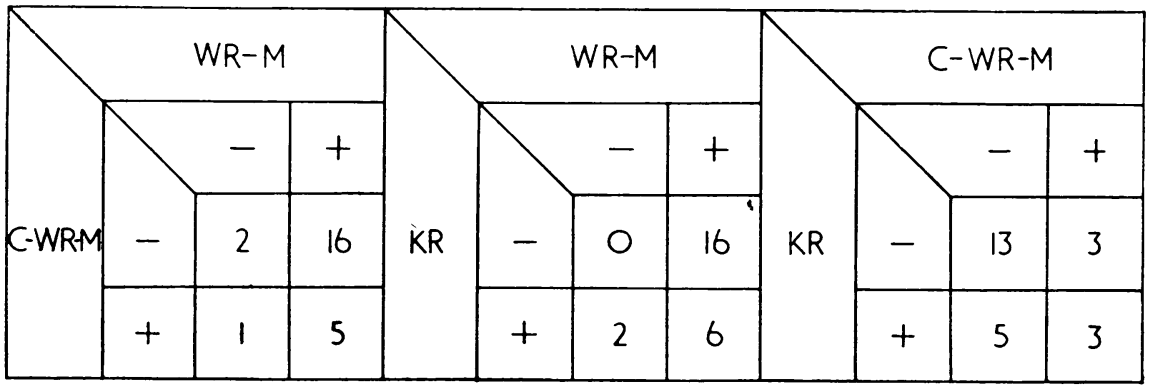


TABLE III

$2 \times 2$ TABLES" FOR TEN BIOLOGICALLY FALSE POSITIVE SERA FOUND AMONG 1,955 NON-SYPHILITIC PREGNANT WOMEN

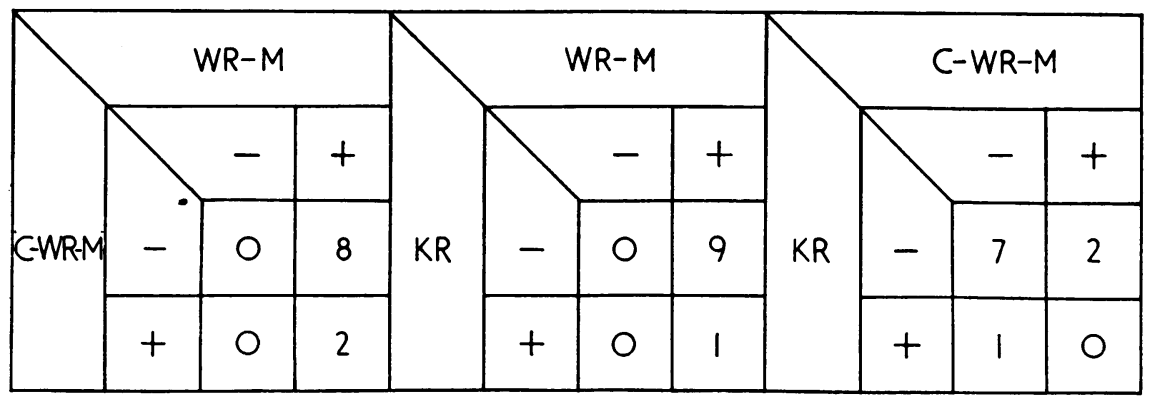

(b) 1,986 sera from pregnant women were examined to find whether the incidence of B.F.P. was higher in them than in "normal" patients. Of the 1,986 sera examined, 1,955 were from patients with neither clinical evidence nor history of syphilis.* The latter figure included ten sera which were biologically false positive in one or more tests, corresponding to a total non-specificity among pregnant women of about 0.5 per cent. The relative incidence of positive reactions in WR-M, C-WR-M, and $K R$ is not significantly different from that found for $(a)$. The results of Penttinen (1946) and of Krag and Røjel (1947) have thus been corroborated, since B.F.P. were not found to be more frequent among pregnant women than among a " mixed" group of patients. All ten biologically false positive sera from pregnant women showed positive reactions by WR-M, corresponding to a non-specificity for WR-M of 0.5 per cent. Two sera were biologically false positive by C-WR-M $(0 \cdot 1$ per cent. $)$ and only one serum was positive by KR ( 0.05 per cent.). By leaving out the WR-M, seven out of ten biologically false positive sera would have been avoided. The relations between the three sero-reactions examined are illustrated in Table III.

WR-M was found to be significantly more frequently positive than either C-WR-M or KR $(P=0.8$ and 0.4 per cent. respectively; no difference in the incidence of B.F.P. could be demonstrated between C-WR-M and KR.

From these observations the following conclusions may be drawn:

* 31 sera (1.6 per cent.) originated from pregnant women with syphilis (clinical and/or annamnestic). Thus, there are significantly fewer syphilitic patients among examined pregnant women than in a group of hospitalized patients, among whom about 10 per cent. were found to be syphilitic (Bang and Krag, 1943 ; Schmidt, 1952).
(1) The non-specificity for pregnant women (about 0.5 per cent.) is not significantly different from the non-specificity for a " mixed" material of patients (about 0.7 per cent.).

(2) WR-M is the reaction that shows the highest non-specificity (about 0.5 per cent.). Among pregnant women, all biologically false positive sera were positive in that test.

(3) WR-M $>$ C-WR-M $=$ KR.

(c) During the test period about 170,000 sera were examined; special attention was devoted to sera from pregnant women and from patients with diseases of the respiratory tract, when such diseases were attended by biologically false positive seroreactions. As already mentioned, this examination was not performed as a parallel examination for the three sero-reactions, because only WR-M and KR were performed as routine tests, but when one or both of these tests showed positive results in screen tests, the sera were examined by C-WR-M along with quantitative WR-M and KR. Thus, in this material, information is not available about the sera which were positive in C-WR-M only, but the magnitude of this figure can be estimated on the basis of the findings reported under $(a)$ and $(b)$.

In $(a)$ and $(b)$ one serum positive by C-WR-M only was found out of 34 biologically false positive sera, i.e. a relative incidence of sera positive by C-WR-M only of 3 per cent., the safety margins being $0 \cdot 1$ to 15 per cent.

Patients with Diseases of the Respiratory Tract.During the test period, sera from 75 patients with diseases of the respiratory tract showed B.F.P.*

\footnotetext{
* The specificity was determined on the basis of questionnaires sent to doctors and hospitals and data obtained from the Central Register of Syphilitic Patients (Jersild, 1919 ; Madsen and Krag, 1937 ; Schmidt, 1951).
} 
On the basis of the findings reported in $(c)$ nothing can be said about the incidence of B.F.P. among such patients, because the number from whom blood specimens were submitted for sero-diagnostic examination is not known, and inquiries, even if based on random samples, would involve considerable technical difficulties. Moreover, information could only be obtained of the number of positive blood specimens from hospitalized patients with diseases of the respiratory tract, because blood samples are not often taken from such patients when treated in their own homes.

For the 75 patients with diseases of the respiratory tract, Table IV shows the distribution of seroreactions by WR-M and KR. Only these two tests are directly comparable in this material, since they were performed for all the sera submitted, positive as well as negative.

\section{TABLE IV}

“ $2 \times 2$ TABLE" FOR 75 NON-SPECIFIC POSITIVE SERA FROM PATIENTS WITH DISEASES OF THE RESPIRATORY TRACT

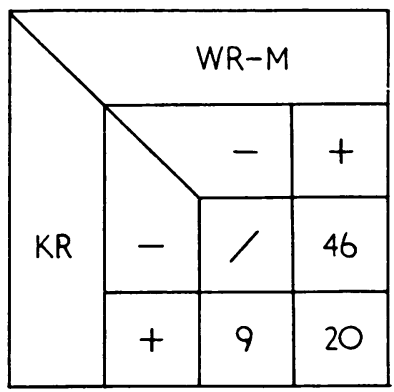

It will be seen that $+/-$ and $-1+$ represent respectively 9 and 46, while the number to be expected if the two tests were to occur in equal positive numbers should be 27 . The figures 9 and 46 are significantly different $(P=<0 \cdot 1$ per cent.), and it has to be accepted that WR-M is considerably more non-specific than KR in sera from patients with diseases of the respiratory tract.

C-WR-M has not been examined on an equal footing with the other tests, because only those sera which were positive in WR-M and/or KR were examined in C-WR-M. A direct comparison of the three tests would therefore not be permissible, and any differences found in specificity would not be a true indication of a higher or lower specificity for C-WR-M, because those sera are missing which are positive in C-WR-M only ; the non-specificity of that test would therefore be found to be unduly low in this selection.

For this material it has therefore been found preferable to compare, for instance, C-WR-M with WR-M under the assumption of $\mathrm{KR}+$, and $\mathrm{C}-\mathrm{WR}$ -
$M$ with KR under the assumption of WR-M+. In a comparison under these conditions, C-WR-M has the same chance of being positive or negative as the test with which it is compared.

Table $\mathrm{V}$ shows $18+/-$ and $7-/+$, whereas the expected numbers are 12 of each kind, if the incidence of positive reactions is equal for the two tests. The figures 18 and 7 are significantly different $(P=4.3$ per cent.) ; hence, it has to be accepted that KR is more non-specific than C-WR-M in the case of WR-M-positive sera from non-syphilitic patients with diseases of the respiratory tract.

TABLE V

“ $2 \times 2$ TABLE" FOR COMPARISON OF KR AND C-WR-M FOR WR-M-POSITIVE SERA FROM NON-SYPHILITIC PATIENTS WITH DISEASES OF THE RESPIRATORY TRACT Assumption : WR-M+

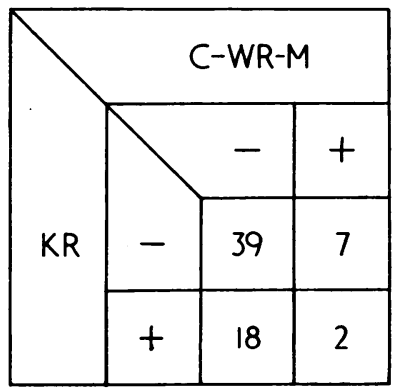

The ratio between the observed figures of $+1-$ and $-/+(18: 7)$ is different from the ratios between the observed figures of $5: 3$, and $2: 1$ respectively, which are found by comparing C-WR-M and KR in the materials $(a)$ and $(b)$. The fact that KR is more non-specific than C-WR-M in this material (consisting entirely of patients with diseases of the respiratory tract) may be explained by one or other of two reasons :

(i) WR-M and KR are more interdependent for sera from patients with diseases of the respiratory tract than WR-M and C-WR-M.

(ii) such sera are more non-specific with $\mathrm{KR}$ than other sera.

WR-M was compared to C-WR-M under analogous conditions, as only cases where $\mathrm{KR}$ was positive were examined (see Table VI, overleaf).

WR-M is significantly more frequently positive than C-WR-M $(P<0.1$ per cent.), and is thus more non-specific than C-WR-M with $\mathrm{KR}+$ sera from patients with diseases of the respiratory tract.

The question whether the three sero-reactions examined here are mutually independent as regards the incidence of B.F.P. is very important. The numbers of C-WR-M-positive and KR-positive sera from patients with diseases of the respiratory 
TABLE VI

" $2 \times 2$ TABLE" FOR COMPARISON OF THE RELATION BETWEEN WR-M TEST AND C-WR-M TEST FOR KR-POSITIVE SERA FROM NON-SYPHILITIC PATIENTS WITH DISEASES OF THE RESPIRATORY TRACT Assumption : $\mathrm{KR}+$

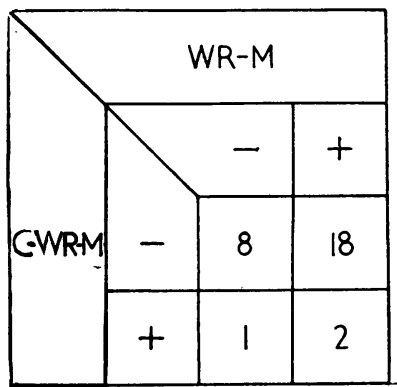

tract (cf. the " $2 \times 2$ Tables ") are so small, however, that the present material cannot be used to test the hypothesis of their being independent.

Figs 1-3 (opposite), showing the quantitative relation of the three sero-reactions, suggest interdependence between WR-M and C-WR-M and between WR-M and KR, while the question of interrelation or not between C-WR-M and KR is still open.

From these observations the following conclusions may be drawn :

(1) For patients with diseases of the respiratory tract the non-specificity of WR-M $>$ KR $>$ C-WR-M,

(2) There is a certain interdependence of titre values for WR-M and positivity of the other tests, positive results for C-WR-M and KR increasing at rising titres of WR-M.

(3) These examinations permit no conclusions to be drawn about the incidence of non-specific positive sero-reactions in patients with diseases of the respiratory tract.

Pregnant Women.-During the test period for (c), 28,735 sera from pregnant women were examined, out of which 126 were B.F.P. in one or more tests. The non-specificity was thus 0.4 per cent.; this conforms to the non-specificity found for the smaller number of pregnant women in examination (b), viz. 0.5 per cent. None of the 126 B.F.P. originated from parturient or aborting patients. The distribution of the positive sera over WR-M and KR is shown in Table VII.

WR-M was found to be significantly more frequently positive than $\mathrm{KR}(P<0 \cdot 1$ per cent.). It is thus established that WR-M is significantly more non-specific among sera from pregnant women than KR. C-WR-M was examined, as was the case for patients with diseases of the respiratory tract, only for those sera which reacted positively
TABLE VII

RELATION BETWEEN WR-M AND KR FOR NON-SPECIFIC POSITIVE SERA FROM NON-SYPHILITIC PREGNANT WOMEN

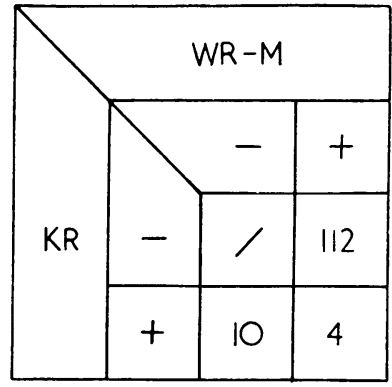

in WR-M and/or KR. An examination of the interrelationship between two tests, assuming positive results in the third test, permits a comparison of C-WR-M with the other tests. On these assumptions " $2 \times 2$ Tables" have been prepared for the comparison of C-WR-M with WR-M, assuming $\mathrm{KR}+$, and with $\mathrm{KR}$ assuming WR-M+ (Tables VIII and IX).

TABLE VIII

INTER-RELATIONSHIP OF WR-M AND C-WR-M FOR KRPOSITIVE SERA FROM 14 NON-SYPHILITIC PREGNANT WOMEN

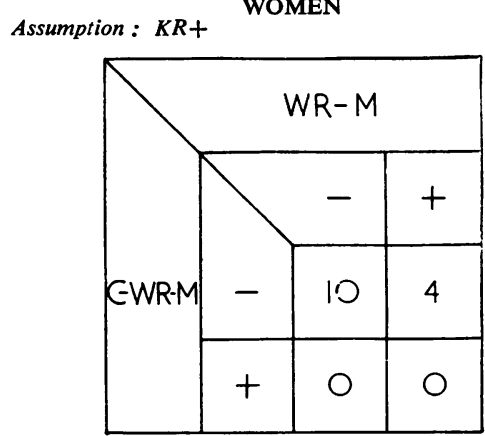

TABLE IX

INTER-RELATIONSHIP OF KR AND C-WR-M FOR WR-MPOSITIVE SERA FROM 116 NON-SYPHILITIC PREGNANT WOMEN

Assumption : $W R-M+$

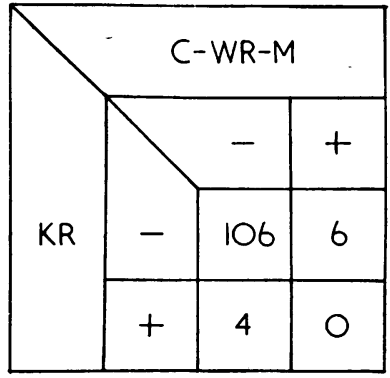




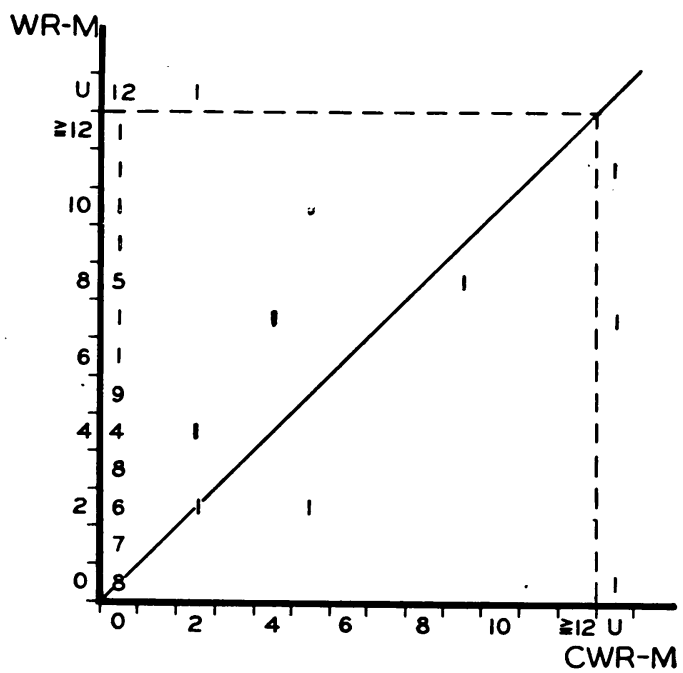

FIG. 1.-Quantitative relation between WR-M and C-WR-M for 75 biologically false positive sera from non-syphilitic patients with diseases of the respiratory tract.

Abscissa : Degrees of potency of C-WR-M. Ordinate: Degrees of potency of WR-M. $\mathbf{U}=$ unreadable reaction.

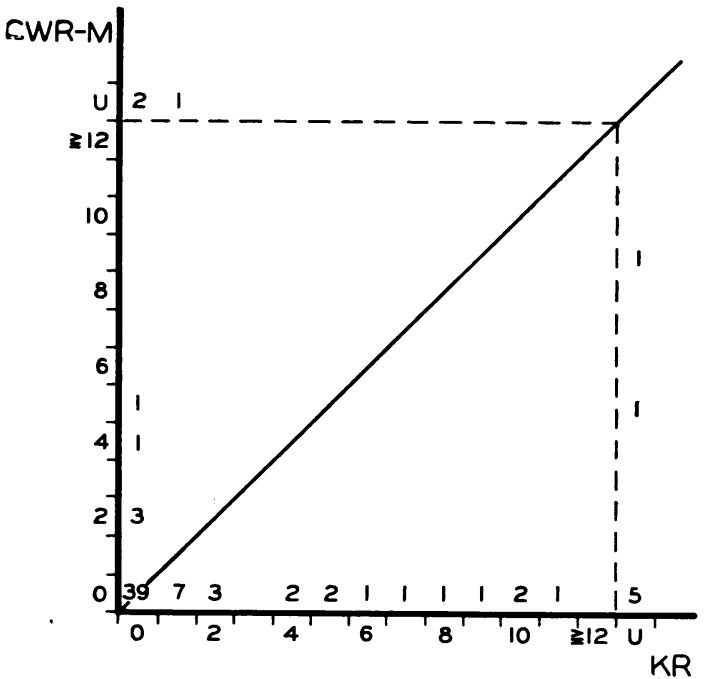

Fig. 3-Quantitative relation between C-WR-M and $K R$ for 75 biologically false positive sera from non-syphilitic patients with diseases of the respiratory tract.

Abscissa: Degrees of potency of KR.

Ordinate: Degrees of potency of C-WR-M.

$\mathrm{U}=$ unreadable reaction.

It will be seen that C-WR-M was not positive in any case of positive $\mathrm{KR}$, but that several positive results were found for C-WR-M where WR-M was positive. By analogy with conditions for patients with diseases of the respiratory tract, the number of pregnant women with B.F.P. in C-WR-M and KR is inadequate to test the hypothesis that these two reactions are independent.

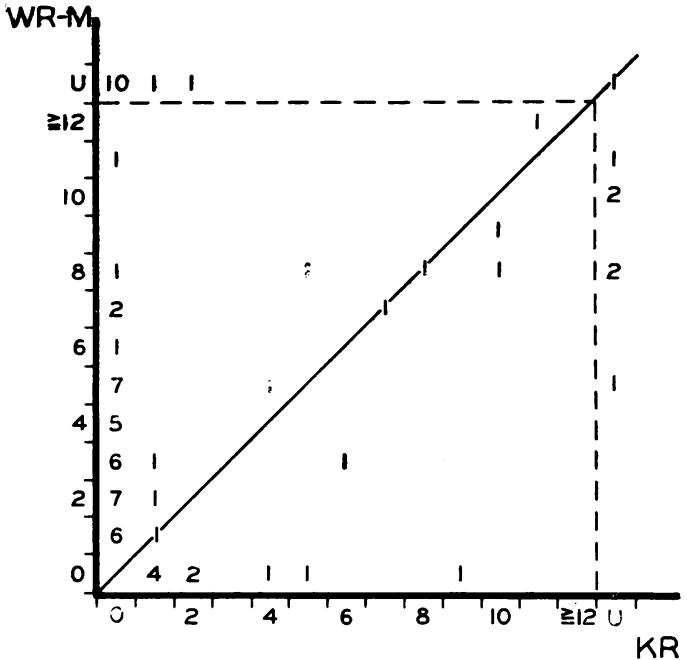

FIG. 2.-Quantitative relation between $\mathrm{WR}-\mathrm{M}$ and $\mathrm{KR}$ for 75 biologically false positive sera from non-syphilitic patients with diseases of the respiratory tract.

Abscissa : Degrees of potency of KR.

Ordinate: Degrees of potency of WR-M.

$\mathbf{U}=$ unreadable reaction.

A quantitative comparison of the three reactions is rendered difficult by the facts that several of the WR-M-positive sera were unreadable, and that very few sera were positive by C-WR-M and KR.

From these examinations the following conclusions may be drawn :

(1) $\mathrm{WR}-\mathrm{M}>\mathrm{KR}$.

(2) $\mathrm{C}-\mathrm{WR}-\mathrm{M}=\mathrm{KR}$.

(3) WR-M cannot be demonstrated to be more non-specific than C-WR-M.*

Unreadable Reactions. - Sera are termed "unreadable" when the reagin content cannot be expressed in degrees of potency because the haemolysis curve is abnormal. The haemolysis curve for an unreadable serum is not " normal " but levelled down, because two or more tubes of the titre range show the same degree of haemolysis, in spite of their different contents of serum. For the sera involved in this examination an anticomplementary effect does not enter into the picture, because the control tube (which contains similar substances as the other tubes, apart from the antigen) shows complete haemolysis.

The present examinations reveal that unreadable reactions in WR-M are considerably more frequent among sera from pregnant women than among sera from patients with infections of the respiratory tract. Thus, among patients with diseases of the respiratory tract there were thirteen unreadable out of $66 \mathrm{WR}-\mathrm{M}$-positive sera (Figs 1 and 2). This is

\footnotetext{
* The number of KR-positive observations is very small.
} 
equal to about 20 per cent. of non-specific positive sera from patients with diseases of the respiratory tract. Further, 73 out of 116 non-specific WR-Mpositive sera from pregnant women were unreadable (i.e. approx. 63 per cent.). The difference between the numbers of unreadable reactions for the two categories of patients is significant $(P<0 \cdot 1$ per cent.). It has to be accepted that WR-M is more frequently unreadable among sera from pregnant women than among sera from patients with diseases of the respiratory tract.

"Unreadability" is generally found among sera showing partial haemolysis in the first two tubes of the titre range, i.e. among sera with a low content of reagin, the two tubes showing equal, or almost equal, percentages of haemolysis although the quantity of serum in the second tube is only onethird that in the preceding tube.

Sera where the highest serum concentration yields about 0 per cent. haemolysis are less frequently unreadable.

As already stated, unreadability is found espec1ally among weakly positive sera ; this is further corroborated by the fact that patients with unreadable reactions are often found to have become spontaneously negative in renewed examinations a short time after the first one. Unreadable reactions, however, are not infrequent at initial formation of reagin in primary syphilis ; unreadability also occurs fairly frequently after antisyphilitic treatment before the serum has become quite negative.

The fact that sera with a low reagin content show the highest incidence of unreadable reactions may explain why the latter are more frequent in pregnant women than in patients with diseases of the respiratory tract, who generally show higher titres of the B.F.P. A comparison of the haemolysis curves for unreadable sera from pregnant women and from patients with diseases of the respiratory tract showed no signs of any difference in " unreadability" for the two categories of patients, the curves being alike.

\section{Discussion}

The ideal antigen for sero-diagnosis of syphilis should be 100 per cent. sensitive and 100 per cent. specific, so that all positive reactions indicate a syphilitic infection, while negative reactions are an unmistakable indication of the absence of active syphilis. It is possible, however, to change the sensitivity of the antigens employed by adjusting the quantities of their different components (Allen and Mason, 1952; Lundbäck, 1952). Antigens can be rendered more specific, for instance, by changing the cardiolipin/lecithin ratio, but the increase in specificity is achieved at the expense of sensitivity. One of the reasons why Price and Wilkinson (1952) found more non-specific reactions with cardiolipin antigen than with unpurified antigen may be that they used cardiolipin and lecithin in the ratio of $1: 1$ in their antigen. The ratio of cardiolipin/lecithin in the author's experiments was $1: 5$.

When sera from patients with diseases of the respiratory tract show a higher incidence of nonspecific positive sero-reactions than sera from other categories of patients, we count such reactions as biologically false positive, though actually they might be classified as technically false positive, because they might be fewer if the composition of antigens was changed.

However, the proportions of cardiolipin, lecithin, and cholesterol in the cardiolipin antigen were so adjusted as to have the maximum sensitivity to syphilitic sera (Maltaner and Maltaner, 1945; Brown, 1948). Because the cardiolipin antigen containing cardiolipin, lecithin, and cholesterol in the respective proportions of $1: 5: 17$ is that which is used in our routine tests, all positive sero-reactions from patients with neither clinical evidence nor history of syphilis are considered in this paper as biologically false positive.

B.F.P. may be a serious problem, among other reasons because it is almost impossible, after institution of treatment, to determine whether the positive reaction indicated a syphilitic infection or whether it was positive for other reasons. Many cases have therefore been kept under treatment and medical control for long periods-the women for each pregnancy.

Several authors have studied the problem of biologically false positive sera; some believe the positive reaction to be a function of the antigen, for instance in the form of impurities in the antigen, while others suggest that the positive reaction might be due to abnormalities in the serum.

Mackie and Anderson (1937) found thus that an acetone-soluble portion of sheep heart, which was discarded during the preparation of antigen, precipitated with sera from both syphilitic and non-syphilitic patients. In their opinion it is possible that biologically false positive sera contain large quantities of a reagin reacting with the impurities of the syphilis antigen.

Widelock and others (1950), in a protracted extraction of beef-heart powder, succeeded in isolating an acetonesoluble antigen which reacted strongly with biologically false positive sera, but weakly, if at all, with sera from syphilitic patients.

Davies (1944) explained biologically false positive sero-reactions as a result of the presence in the positive serum of an antibody or of a reagin either identical to, 
or in certain ways different from, syphilitic reagin, but cross-reactive with the unpurified antigen or components thereof.

Neurath and others (1947 $a$ and $b$ ) fractionated syphilitic sera into serologically active globulin fractions, G I, G II, and G III ; they found that the strongest serological activity of the protein substances precipitated at the smallest concentration of ammonium sulphate, viz. G I and G II. The serum component which precipitated at the stronger concentration of ammonium sulphate, G III, showed little or no serological activity. The unprecipitated unpurified albumin fraction, still in solution, was serologically inactive.

In examinations of sera from patients with B.F.P., the above-mentioned authors found that about one-half of the examined sera showed stronger serological activity of the fractions G I and G II than of unfractionated serum, possibly because the discarded fractions (G III and the albumin fraction) contained substances which inhibited the reaction. They later succeeded in isolating from sera a thermostable substance which inhibited the flocculation of lipoidal antigens in the euglobulin fraction of biologically false positive sera, but this thermostable substance did not affect the flocculation of lipoidal antigens in the euglobulin fraction of syphilitic sera.* Chemically, this inhibitory substance, which was demonstrable in about one-half of the persons examined, proved to be a lipoprotein, and electrophoresis showed it to belong to the $\alpha$-globulin fraction.

On the strength of their experiments, Neurath and others (1947a and b) advanced the theory that there are two separate and chemically different antibodies which cause positive sero-reactions.

(i) the syphilitic antibody,

(ii) the biologically false positive antibody.

Volkin (1949) demonstrated the inhibitory substance in serum to be lecithin, the solubility conditions and the $N / P$ ratio for the inhibitory substance being the same as for human serum lecithin. Certain conditions in the fractionation of the inhibitory substance, $\dagger$ which appeared to be at variance with its supposed lipoidal character, are explained by the fact that the lecithin is in fixed combination with protein.

Volkin further demonstrated that egg lecithin was devoid of inhibitory effect, while beef-heart lecithin was at least as inhibitory as human lecithin.

Meinicke (1952) succeeded in changing presumptive B.F.P. into negative reactions in undernourished patients by oral administration of lecithin ; he also managed to render the Meinicke antigen less sensitive by adding concentrates of lipoid to the original extract.

Lindau and Laurell (1952), after experiments with paper electrophoresis of biologically false positive sera, have

\footnotetext{
* Neurath and others (1947a and b) used cardiolipin antigen for their experiments, finding that inhibition was more specific to antigens of high chemical purity than to more sensitive but less specific antigens (e.g. Kahn's presumptive test).

t The fraction cannot be isolated from IV-1 protein with ether, and cannot be removed by dialysis or by heat coagulation of the IV-1 protein.
}

advanced the theory that the false positive reagins are present as a normal component of serum, but clinically inactive, because they are in a state of equilibrium with the inhibitory factor described by Neurath. If this equilibrium is disturbed, the false positive reagins become manifest through the positive reaction. In seventeen WR-negative normal sera, Lindau and Laurell could (by concentration of serum) produce positive WR with unpurified antigen in the front part of the $\gamma$-globulin fraction, while WR with cardiolipin was negative. They succeeded, however, in producing a positive WR with cardiolipin antigen also, by using ten times the normal amount of cardiolipin antigen.

$\operatorname{Kahn}(1949,1951)$, by demonstrating universal biologic reactions, supplied the theory that this potential production of biologically false positive reactions is a latent quality in many sera ; using Kahn antigen in different saline concentrations and at different temperatures, he was able to produce flocculation in nearly all sera.

D'Alessandro (1946) demonstrated three different antibodies in syphilitic sera, viz. one anti-lipoidal and two anti-treponemal. Of the anti-treponemal antibodies, one was thermolabile while the other was thermostable. According to this author, who performed his experiments with "palligen", only the anti-treponemal antibodies occur early in the primary stage of syphilis, while in the secondary and tertiary stages, the anti-lipoidal and the anti-treponemal antibodies occur together.

Schmidt (1952) from his own observations believes that syphilitic serum contains at least two different reagins. He has tried to prove this through the changed interrelationship between unpurified antigen and cardiolipin antigen at different clinical stages of syphilis, S I and S III.

Nielsen and Schmidt (1953) found by observation of the sero-reactions in rabbits infected with Nicol's pathogenic spirochaetae that the treponemal immobilization test, T.P.I.50-18 (Nelson and Mayer, 1949) becomes positive somewhat later than the sero-reactions with unpurified antigen and cardiolipin antigen.

Durel and others (1952) are among other authors who have reported that in untreated patients with fresh infections the T.P.I. does not give as dependable results as the "old" sero-reactions. Thus, it appears that the anti-lipoidal antibodies, demonstrated with antigens produced from alcoholic tissue extracts (or with cardiolipin alone (Lundbäck, 1952)), occur earlier than the treponemal-immobilizing antibody.

This digression from biologically false positive reactions to the serological aspects of syphilis has been made because the courses of the different reactions (WR-M and C-WR-M) show features which appear to be identical for sera with biologically false positive reactions and for sera from patients with clinically diagnosed syphilis. Thus, WR-M, which is very sensitive in primary syphilis and is generally the first sero-reaction to become 
positive after infection, also shows the largest number of positive reactions in patients without other evidence of syphilis.

The Wassermann antigen, as modified by Mørch, and the cardiolipin antigen are made up of the same components, viz. cardiolipin, lecithin, and cholesterol, though in mutually different ratios. In addition, the Wassermann antigen contains some unknown impurities, possibly lipo-proteins. These unknown substances presumably cause the higher reactivity of WR-M to sera from patients with primary syphilis and to biologically false positive sera. The anti-lipoidal antibody present in syphilitic sera may be identical with, or merely cross-reactive with, the antibody which is present in biologically false positive sera. Biologically false positive seroreactions could conceivably occur mainly in diseases where the serum contains an anti-lipoidal antibody, possibly analogous with the conditions existing in primary syphilis.

It is remarkable that diseases connected with tissue decay are particularly responsible for false positive reactions. The destruction of tissue may start a production of reagins, the tissue products acting as haptens, possibly supplemented by protein substances to form full-antigens.

Sartorius (1951) points out that the false positive sero-reactions are more frequent in early acute cases of malaria where considerable destruction of erythrocytes takes place follow'ed by formation of antibodies, while chronic malaria is less frequently attended by false positive reactions.

Mohr and Moore (1952) also found more frequent false positive reactions in diseases involving decay of tissue. Among 51 patients with false positive reactions, 31 had definite "collagen disease", while another fourteen, who were clinically healthy, had changes in their blood suggesting early lupus erythematosus.

In Denmark, infection of the respiratory tract is the most frequent cause of false positive seroreactions; in the present work special attention has therefore been devoted to the serological conditions existing for this group of patients.

The positive reactions seen in pneumonic diseases seem to be connected closely with the disease itself, since the positive reaction generally occurs shortly after the infection (perhaps concurrently with the incipient formation of antibodies against the infecting micro-organisms?) and recedes shortly after recovery from the disease (Krag and Lønberg, 1938 ; Reyn, 1941) (perhaps because of decreasing concentration of antibody ?). Changes in the serum content of lipoproteins may also influence the occurrence of biologically false positive reactions.
Electrophoresis of sera from patients with acute infections have shown a frequent rise of the serum content of $\alpha$-globulins.

Blix (1939) examined sera from patients with pneumococcal pneumonia and found, in addition to a hypalbuminaemia, a relative and absolute increase of $\alpha$-globulins, while $\beta$-and $\gamma$-globulins were essentially unchanged.

Malmros and Blix (1946) observed similar, though less pronounced, reactions in acute tonsillitis, changed globulin conditions being demonstrable in serum for 2-3 weeks after the outbreak of the disease.

Benhamou (1947) reported an increase in the globulin content of the serum in typhus abdominalis, accompanied by a decrease in albumin concentration. The increase in globulin content comprised all globulin fractions. Clinical recovery was found to be connected with a decline in $\alpha$-globulin and a rise in the $\gamma$-globulin content.

The changed serological conditions in acute infections causing decay of tissue are presumably due to a release of nuclein acids during cellular destruction. The inhibitor normally present may be destroyed, upsetting a balance existing in normal cases between the inhibitor and a " latent "antibody.

However, the rise in the $\alpha$-globulin content of serum cannot alone be responsible for biologically false positive reactions since a constant increase in $\alpha$ - and $\beta$-globulins occurs throughout pregnancy where the $\gamma$-globulins are unchanged. The incidence of biologically false positive reactions in pregnant women is of the same magnitude as in a mixed group of patients (Schmidt and Reyn, 1952).

The present examinations have shown that complement-fixation tests performed with unpurified antigen (WR-M) show substantially more frequent non-specific positive results in diseases of the respiratory tract than the complement-fixation test performed with cardiolipin (C-WR-M). However, some interdependence seems to exist between the two tests, the C-WR-M showing increasingly positive results for rising titres in the WR-M.

The frequent recording of biologically false positive reactions in pregnant women is probably due to the fact that pregnant women are examined in larger numbers than other groups of patients. This makes pregnant women the group of patients that has the highest number of non-specific seroreactions without having a relative increase in the incidence of biologically false positive sero-reactions, as also demonstrated in this paper. Generally, non-specific reactions in pregnant women are of longer duration and often persist after delivery, which would seem to suggest that the positive 
reaction is a property existing in the serum of the woman, rather than a result of the pregnancy.

Only large-scale examinations of different groups of patients may reveal those diseases which show particularly high incidences of B.F.P., and may also indicate a non-specificity of the individual seroreactions for each group of diseases.

\section{Summary and Conclusions}

(1) The purpose of this study was to determine and compare the specificity of the Wassermann complement-fixation test, as modified by Mørch, performed with unpurified antigen (WR-M) and with cardiolipin (C-WR-M) composed of cardiolipin, lecithin, and cholesterol in proportions of $1: 5: 17$. At the same time, the specificity of Kahn's standard reaction (KR) was compared with that of the complement-fixation tests.

(2) The study was divided into three parts. Parallel examinations, using WR-M, C-WR-M, and $\mathrm{KR}$ were performed for

(a) 3,565 mainly hospitalized, non-syphilitic patients ;

(b) 1,955 non-syphilitic pregnant women ;

(c) about 170,000 sera submitted during a 4 months' test period to the Sero-diagnostic Department, Statens Seruminstitut. All the blood samples were examined routinely by WR-M and KR. Sera showing positive reactions in either or both of these tests were examined by C-WR-M. As a result, details are missing on those sera which were positive only in C-WR-M, but the magnitude of that figure is known from groups $(a)$ and $(b)$.

(3) The number of non-specific reactions corresponds to a non-specificity for $(a)$ of about 0.7 per cent. and for $(b)$ of about 0.5 per cent. These figures are not significantly different; hence it must be accepted that biologically false positive sero-reactions do not occur more frequently among pregnant women (b) than among " normal" patients $(a)$.

(4) WR-M is the most non-specific sero-reaction both for $(a)$ and $(b)$, being significantly more frequently positive than C-WR-M and KR. No difference in specificity could be demonstrated between C-WR-M and KR for groups $(a)$ and $(b)$.

(5) Among patients with diseases of the respiratory tract showing biologically false positive reactions, WR-M was significantly more nonspecific than KR. C-WR-M was not directly comparable with WR-M and KR, because it was performed only if either or both of the latter reactions were positive. The C-WR-M was therefore compared with $\mathrm{KR}$ for those sera which were positive in WR-M, and with WR-M for those sera which were positive in KR. On these assumptions,
$\mathrm{KR}$ as well as WR-M proved to be significantly more frequently positive than C-WR-M, thus being more non-specific to sera from patients with diseases of the respiratory tract. A certain interdependence appears to exist between the degree of potency of WR-M and the positivity of C-WR-M and KR respectively (rising numbers of positive reactions with C-WR-M and KR respectively, at increasing titres of WR-M), while inadequate numbers of $\mathrm{C}-\mathrm{WR}-\mathrm{M}+$ and $\mathrm{KR}+$ sera prevented determination of a potential interdependence or independence of these two sero-reactions.

(6) Among sera from pregnant women with biologically false positive reactions, WR-M was significantly more non-specific than KR. C-WR-M was compared with WR-M and KR on the assumptions explained above in Item 5 . The small number of KR-positive sera (14), for which C-WR-M and WR-M were compared, does not allow us to decide whether significant differences in specificity exist between the two complement-fixation tests, but in this small sample of sera there is nothing to indicate that the relation between C-WR-M and WR-M should differ from that determined for group (b). No differences in specificity could be demonstrated between C-WR-M and KR when their specificities for WR-M - sera were compared.

(7) The incidence of unreadable reactions in WR-M was significantly more frequent among sera from pregnant women than among sera from patients with diseases of the respiratory tract. No differences were observed in the haemolysis curves for the unreadable sera from the two categories of patients.

(8) The relation between C-WR-M and WR-M among sera with biologically false positive reactions is suggestive of the relation between the two complement-fixation tests in primary syphilis. It is suggested that the serum changes causing biologically false positive sero-reactions may be very largely analogous to those occurring in primary syphilis.

\section{REFERENCES}

D'Alessandro, G. (1946). Boll. Ist. sieroter. milan., 25, 138.

Allen, R. H., and Mason, M. A. (1952). Canad. J. publ. Hlth., 43, 128. Bang, O., and Krag, P. (1943). Ugeskr. Laeg., 105, 203.

Benhamou (1947). Cited in "Die quantitative Elektrophorese in der Medizin "'. Springer, Berlin (1952).

Blix (1939). Ibid.

Brown, R. (1948). Amer. J. clin. Path., 18, 565.

Davis, B. D. (1944). Medicine, Baltimore, 23, 359.

Durel, P., Sausse, A., and Borel, L. J. (1952). British Journal of Venereal Diseases, 28, 68.

Eldh, S. M. (1932). Svenska LäkT., 29, 373.

Hecht, H. (1952). Ohio St. med. J., 48, 213.

Jersild, O. (1919). Hospitals tidende, 62, 382.

Kahn, R. L. (1949). Amer. J. clin. Path., 19, 347.

(1951). Univ. Mich. med. Bull., 17, 217. 
Krag, P., and Lønberg, A. (1938). Ugeskr. Laeg., 100, 497.

—— and Røjel, J. (1947). Ibid., 109, 493.

Lindau, A. (1939). Acta chir. scand., 82, 355.

$\longrightarrow$, and Laurell, A. B. (1952). Personal communication.

Lundbäck, H. (1952). Thesis, Uppsala.

Mackie, T. J., and Anderson, C. G. (1937). J. Path. Bact., 44, 603.

Madsen, T., and Krag, P. (1937). Acta derm.-venereol., Stockh., 18, 546 .

Malmros and Blix (1946). Cited in " Die quantitative Elektrophorese in der Medizin". Springer, Berlin (1952).

Maltaner, E., and Maltaner, F. (1945). J. Immunol., 51, 195.

Meinicke, K. (1952). Wien. med. Wschr., 102, 378.

Mohr, C. F., and Moore, J. E. (1952). "Recent Advances in the Study of Venereal Diseases, A Symposium."

Nelson, R. A., and Mayer, M. M. (1949). J. exp. Med., 89, 369.

Neurath, H., Volkin, E., Craig, H. W., and Erickson, J. O. (1947a). Amer. J. Syph., 31, 436.

- - Erickson, J. O., Craig, H. W., Putnam, F. W., and Cooper, G. R. (1947b). Ibid., 31, 347 .

Nielsen, H. A., and Schmidt, H. Unpublished observations.
Penttinen, K. (1947). Acta obstet. gynec. Scand., 27, Suppl. 3.

Price, I. N. Orpwood, and Wilkinson, A. E. (1952). British Journal of Venereal Diseases, 28, 16.

Rein, C. R., and Bossak, H. N. (1946). Amer. J. Syph., 30, 40.

, and Elsberg, E. S. (1945). J. invest. Derm., 6, 113.

, and Kent, J. F. (1947). J. Amer. med. Ass., 133, 1001

Reyn, A. (1941). Ugeskr. Laeg., 103, 137.

$\longrightarrow$, and Schmidt, H. (1951). Ugeskr. Laeg., 113, 1427.

Sartorius, F. (1951). Z. ärtzl. Fortbild., 45, 154

Schmidt, H. (1951). British Journal of Venereal Diseases, 27, 23. (1952). Ibid., 28, 169.

_- and Reyn, A. (1952). Ugeskr. Laeg., 114, 1485.

Turner, T. B. (1952). “First International Symposium on Yaws Control ", p. 15. Bangkok, Thailand. WHO/VD/ 104, Sept., 1952.

Vogelsang, T. M. (1948). Acta path. microbiol. scand., 25, 167.

Volkin, E. (1949). J. Immunol., 61, 143.

Widelock, D., Gonshorek, M. F., and Marsden, L. (1950). J. vener. Dis. Inform., 31, 133.

Wiingaard, P. (1948). Thesis, Copenhagen. (Eng. summary.) 